\title{
ATRIBUTOS FÍSICOS DO SOLO EM SISTEMAS DE MANEJO DE SOLO E DE ROTAÇÃO DE CULTURAS $\left({ }^{1}\right)$
}

\author{
SILVIO TULIO SPERA $\left(\left(^{2 *}, 3\right)\right.$; HENRIQUE PEREIRA DOS SANTOS $\left({ }^{3}\right)$; $\operatorname{GILBERTO~OMAR~TOMM~}\left({ }^{3}\right)$; \\ RAINOLDO ALBERTO KOCHHANN $\left({ }^{3}\right)$; ALEXANDRE ÁVILA $\left({ }^{4}\right)$
}

\begin{abstract}
RESUMO
A compreensão e a quantificação do impacto do uso e manejo do solo na qualidade física são fundamentais no desenvolvimento de sistemas agrícolas sustentáveis. Com o objetivo de avaliar o efeito de sistemas de manejo de solo e de rotação de culturas sobre alguns atributos físicos de solo em um experimento instalado em 1985, em Latossolo Vermelho Distrófico típico argiloso, em Passo Fundo (RS), estudou-se a variação desses atributos entre 2001 e 2005. Os tratamentos consistiram de quatro modos de manejo de solo: 1) plantio direto (PD); 2) cultivo mínimo (CM); 3) preparo convencional de solo com arado e grade de discos (PCD) e 4) preparo convencional de solo com arado de aivecas e grade de discos (PCA), e três sistemas de rotação de culturas: I (trigo/soja), II (trigo/soja e ervilhaca/milho ou sorgo) e III (trigo/soja, aveia branca/soja e ervilhaca/milho ou sorgo). O delineamento experimental foi em blocos completos ao acaso, com parcelas subdivididas e três repetições. A parcela foi constituída pelos sistemas de manejo de solo, e as subparcelas, pelos sistemas de rotação de culturas. Amostras de solo também foram coletadas em um fragmento de floresta subtropical adjacente ao experimento, como testemunha da condição original do solo. Os sistemas de manejo e de rotação de culturas influenciaram os atributos físicos do solo quando comparados entre si e com a condição sob floresta subtropical. Em quatro anos (2001 a 2005) ocorreram alterações significativas nos atributos físicos dos solos entre os distintos tipos de manejo. No sistema PD houve maior densidade de solo e microporosidade em comparação aos demais, principalmente na camada subsuperficial, e os sistemas de rotação de plantas não afetaram positivamente os atributos físicos de solo independentemente do tipo de manejo.
\end{abstract}

Palavras-chave: plantio direto, densidade de solo, porosidade total, microporosidade, macroporosidade.

\section{ABSTRACT \\ SOIL TILLAGE AND CROP ROTATION SYSTEMS ON THE SOIL PHYSICAL ATTRIBUTES}

Evaluation and quantification of soil use impact on its physical quality are important to achieve sustainable cropping systems. Soil physical attributes were assessed after twenty years of implementation (1985 to 2005) on a typical Dystrophic Red Latosol (Rodic Haplidox) located in Passo Fundo, Rio Grande do Sul State, Brazil. Four soil tillage systems were evaluated: 1) no-tillage; 2) minimum tillage; 3) conventional tillage using a disk plow plus disk harrow, and 4) conventional tillage using a moldboard plow plus disk harrow. Three crop rotation systems were evaluated: I (wheat/soybean), II (wheat/soybean and common vetch/corn or sorghum), and III (wheat/soybean, common vetch/corn or sorghum and white oats/soybean). A randomized complete block design, with split-plots and three replicates, was

( $\left.{ }^{1}\right)$ Recebido para publicação em 2 de abril de 2007 e aceito em 18 de junho de 2009.

$\left(^{2}\right)$ Embrapa Trigo, Caixa Postal 451, 99001-970 e aluno do PPGAgro - Universidade de Passo Fundo, Caixa Postal 611, 99001-970 Passo Fundo (RS). E-mail: spera@cnpt.embrapa.br $\left(^{*}\right)$ Autor correspondente.

$\left({ }^{3}\right)$ Embrapa Trigo, Passo Fundo (RS). Bolsista do CNPq. PQ. E-mail: hpsantos@cnpt.embrapa.br; tomm@cnpt.embrapa.br; rainoldo@cnpt.embrapa.br.

$\left({ }^{4}\right)$ Acadêmico de Agronomia da Universidade de Passo Fundo - Faculdade de Agronomia e Medicina Veterinária, Passo Fundo (RS). Bolsista CNPq-IC. 
used. The main plots were formed by the soil tillage systems, while split-plots were composed by crop rotation systems. As control, soil samples were collected in a subtropical forest fragment adjacent to the experiment. Soil tillage systems and crop rotation systems influenced soil physical parameters when compared to the samples collected in a fragment of subtropical forest. During four years (2001 to 2005) the physical attributes were altered among different soil tillage management. The no-tillage system (PD) presented higher soil bulk density and microporosity in comparison to the other systems, mainly in 0.10 to $0.15 \mathrm{~m}$ layer, characterizing high soil compaction levels. Crop systems did not promote favorable changes in physical attributes independent of the type of soil management.

Key words: no-tillage, soil bulk density, total soil porosity, soil microporosity, soil macroporosity.

\section{INTRODUÇÃO}

A tentativa de reverter o processo de degradação física do solo e de recuperar a capacidade produtiva do solo tem conduzido a uma mudança de postura por parte dos agentes envolvidos no processo produtivo, especialmente na região Sul do Brasil (AITA e Giacomini, 2006). Um exemplo desta mudança é a adoção do sistema plantio direto e a rápida evolução do mesmo nas duas últimas décadas, tanto em grandes como em pequenas propriedades rurais.

Com a substituição gradativa do preparo convencional de solo mediante revolvimento por arados e grades, pelo sistema plantio direto, nos últimos anos, ocorreram mudanças na qualidade do solo. A degradação do solo causada pela perda de condições favoráveis ao desenvolvimento vegetal pode reduzir sua capacidade produtiva (Albuquerque et al., 1995). Sistemas de manejo adequados de solo sob rotação de culturas devem amenizar esses problemas e agir no sentido de restaurar a estrutura do solo. Para avaliação da estrutura do solo, os atributos densidade de solo, porosidade total, macro e microporosidade ainda são indicadores utilizados (REICHERT et al., 2003).

De acordo com Arshad et al. (1996), a densidade do solo acima de $1,40 \mathrm{Mg} \mathrm{m}^{-3}$ para solos argilosos é restritiva ao crescimento radicular e, consequentemente ao rendimento de grãos das espécies cultivadas. Uma série de trabalhos tem registrado, sob plantio direto, valores maiores de densidade do solo e de resistência à penetração, e menores de porosidade total e de macroporosidade na camada superficial, quando comparados aos sistemas de preparo convencional de solo (DA Ros et al., 1997; Albuquerque et al., 2001; Beutler et al., 2001; Stone e SilveIRA, 2001). De acordo com alguns autores, esse problema pode estar ocorrendo devido ao trânsito de máquinas em solos argilosos em condição de elevada umidade (Stone e Silveira, 2001; Oliveira et al. 2004). De acordo com Costa et al. (2003), SPERA et al. (2004) e MARColan e Anghinoni (2006), maior densidade de solo observada, sob plantio direto, entretanto, não tem afetado o rendimento de grãos das culturas, sendo compensada por outras melhorias da qualidade física do solo. Por outro lado, o efeito do sistema de cultivo (rotação ou sucessão de culturas) tem sido pouco eficiente, em relação ao efeito do sistema de manejo de solo, em modificar os atributos físicos de solo (BERTOL et al., 2004).

O monitoramento da qualidade do solo por meio dos atributos físicos é importante para a manutenção e avaliação da sustentabilidade dos sistemas agrícolas. Esse monitoramento pode ser feito por meio de experimentos de longa duração sob sistemas de manejo de solo e de rotação de culturas (REICHERT et al., 2003).

O presente estudo teve como objetivo avaliar o efeito de sistemas de manejo de solo e de rotação de culturas sobre alguns atributos físicos de solo, em um período de quatro anos, em ensaio de longa duração.

\section{MATERIAL E MÉTODOS}

O ensaio foi desenvolvido em área experimental da Embrapa Trigo, em Passo Fundo (RS), desde 1985, em Latossolo Vermelho Distrófico típico argiloso (STRECK et al., 2002), cujas avaliações dos atributos físicos de solo foram realizadas em 2001 e em 2005. O delineamento experimental foi o de blocos ao acaso, com parcelas subdivididas e três repetições. A parcela principal foi constituída pelos sistemas de manejo de solo. A dimensão de cada parcela foi $4 \times 90 \mathrm{~m}$ e das subparcelas (sistemas de rotação de culturas) de $4 \times 10 \mathrm{~m}$. Os tratamentos foram constituídos por quatro sistemas de manejo de solo (SMSs): 1) plantio direto (PD), 2) preparo de solo com equipamento de hastes para cultivo mínimo escarificador por meio de equipamento de hastes rígidas $\mathrm{Jumbo}^{\circledR}(\mathrm{CM})$, 3) preparo convencional de solo com arado de discos mais grade de discos (PCD) e 4) preparo convencional de solo com arado de aivecas mais grade de discos (PCA); e por três sistemas de rotação de culturas (SRCs): I (trigo/soja), II (trigo/soja e ervilhaca/milho ou sorgo) e III (trigo/soja, aveia branca/soja e ervilhaca/ milho ou sorgo). Um fragmento de floresta subtropical com araucárias, adjacente ao experimento, também foi amostrado, com o mesmo número de repetições, e usado como um referencial do estado estrutural do solo anterior às alterações promovidas pelo uso agrícola. 
Em novembro de 1985, antes da semeadura das culturas de inverno para instalação do experimento, a camada de solo de 0 a $0,20 \mathrm{~m}$ foi amostrada (amostras compostas) em cada parcela conforme CFS-NRS (1995) e os resultados das análises indicaram níveis de matéria orgânica e de nutrientes considerados adequados, porém com acidez elevada. Esses dados estão apresentados em SANTOS e Tomm (2003). O solo foi revolvido por meio de arado e gradeado para a incorporação de $7,0 \mathrm{t} \mathrm{ha}^{-1}$ de calcário dolomítico (PRNT 90 \%), visando a elevar o pH em água a 6,0 (CFS-NRS, 1995). O calcário foi aplicado em duas vezes: metade antes da aração (arado de discos) e metade antecedendo a gradagem (grade niveladora de discos), conforme recomendação de Denardin e KochHann (1993). A adubação de manutenção foi baseada na média dos valores observados em análises químicas da área experimental, conforme CFS-NRS (1995).

Em agosto de 2001 e de 2005, após a colheita das culturas de inverno, foram coletadas amostras não deformadas de solo nas camadas de 0 a 0,05 e de 0,10 a 0,15 m, para determinar a densidade de solo e a porosidade total utilizando-se anéis volumétricos de cerca de $207 \mathrm{~cm}^{3}$. A microporosidade foi considerada como conteúdo volumétrico de água equilibrada na mesa de tensão a 0,60 $\mathrm{m}$ de coluna de água e a macroporosidade calculada por diferença entre a porosidade total e a microporosidade. Esses métodos constam em EMBRAPA (1997).

Os sistemas de manejo de solo foram comparados para cada atributo físico do solo, em cada profundidade de amostragem. As profundidades de amostragem de solo foram comparadas em um mesmo sistema de manejo de solo. Todas as comparações dentro de cada ano foram realizadas por meio de contrastes de interesse não ortogonais de um conjunto de $\mathrm{t}-1$ contrastes com um grau de liberdade, conforme STEEL e TORRIE (1980), para permitir que se enfatize o efeito ano (CADY, 1991). A significância dos contrastes, dentro de cada ano, foi dada pelo teste F, levando-se em conta o desdobramento dos graus de liberdade do erro. As médias dos resultados das avaliações de 2001 e 2005 foram comparadas por meio de contrastes, enquanto, entre 2001 e 2005, foi realizada a comparação de cada atributo pelo teste de Duncan (SAS, 2003).

\section{RESULTADOS E DISCUSSÃO}

A análise estatística revelou que não houve interação entre os tipos de manejo de solo e os de rotação de culturas, porém constatou diferenças nos atributos entre os sistemas de manejo de solo e de plantas.
Sistemas de manejo de solo - A densidade de solo, em todos os sistemas de manejo de solo (SMS), em 2005, revelou valores menores, em comparação ao verificado em 2001, nas camadas de 0 a 0,05 m e de 0,10 a $0,15 \mathrm{~m}$, após quatro anos de cultivo (Tabelas 1 , 2 e 3). Secco et al. (2005) obtiveram, na camada de 0 a $0,07, m$ resultados que corroboram com tal constatação, em três anos de estudo, somente para PD. Houve diferença entre os valores de densidade de solo dos SMSs apenas na camada de 0,10 a 0,15 m. Em 2005, em PD houve maior densidade de solo do que nos demais SMSs estudados indicando a presença de camada compactada a partir dos $0,10 \mathrm{~m}$ de profundidade nos tipos de preparo convencional de solo, em contraste à compactação relativamente uniforme em toda a profundidade estudada sob PD (Bertol et al. 2004). A densidade observada na floresta subtropical (FST), que preserva a condição estrutural original do solo, permite constatar que o uso do solo promoveu alterações que indicam degradação da estrutura do solo independentemente do SMS estudado, em ambas as camadas avaliadas. Resultados semelhantes foram observados por ANJos et al. (1994), em quatro tipos de solo, de quatro municípios dos Estados de Santa Catarina, nos quais, as médias desses solos, sob PCD e sob PD, indicaram maior densidade de solo do que em floresta, na camada de 0 a $0,20 \mathrm{~m}$.

$\mathrm{Na}$ camada superficial, os valores da densidade de solo foram estatisticamente semelhantes em todos os SMS, tanto em 2001 (Tabela 2) como em 2005 (Tabela 3), entretanto, os valores desse ano foram menores. LLANILLO et al. (2006) atribuíram a ocorrência de menores valores de densidade do solo na camada superficial do sistema plantio direto ao efeito do aumento no nível de matéria orgânica. Em 1993, o nível de matéria orgânica do solo, nesse experimento, era maior no PD (38 $\left.\mathrm{g} \mathrm{kg}^{-1}\right)$, em relação ao PCD (31 g $\left.\mathrm{kg}^{-1}\right)$ e ao PCA $\left(29 \mathrm{~g} \mathrm{~kg}^{-1}\right)$, na camada de 0 a $0,5 \mathrm{~m}$ (Santos e Tomm, 2003). Oliveira et al. (2004) observaram densidade de solo sob PD inferior ou igual àquela sob PCD, possivelmente em razão do maior teor de C presente no solo e ausência de revolvimento. Estas condições são semelhantes ao do experimento deste trabalho. AlBUQUERQUE et al. (1995), em Latossolo Vermelho-Escuro e Costa et al. (2003), em Latossolo Bruno, também não observaram aumento da densidade do solo no sistema PD, em comparação ao PCD.

Os valores de densidade de solo, entre as profundidades avaliadas de todos os SMSs (Tabelas 1,2 e 4) foram menores na camada de 0 a $0,05 \mathrm{~m}$ do que na camada de 0,10 a $0,15 \mathrm{~m}$. Resultados concordantes foram observados por TREIN et al. (1991) e por DA Ros et al. (1997). 
Tabela 1. Valores de densidade de solo, porosidade total, microporosidade e macroporosidade, nas camadas de $0-0,5$ m e 0,10-0,15 m, determinado após as culturas de inverno, em quatro sistemas de manejos de solo e três rotações de culturas, em 2001 e 2005

Sistemas de manejo de solo e rotação de culturas Camada $(\mathrm{m})$



\section{Plantio direto}

Preparo convencional de solo com arado de discos

Preparo convencional de solo com arado de aivecas Cultivo mínimo

Floresta subtropical

Plantio direto

Preparo convencional de solo com arado de discos

Preparo convencional de solo com arado de aivecas Cultivo mínimo

Floresta subtropical

Plantio direto

Preparo convencional de solo com arado de discos

Preparo convencional de solo com arado de aivecas Cultivo mínimo

Floresta subtropical

Plantio direto

Preparo convencional de solo com arado de discos

Preparo convencional de solo com arado de aivecas

Cultivo mínimo

Floresta subtropical

Rotação de culturas I

Rotação de culturas II

Rotação de culturas III

Floresta subtropical

Rotação de culturas I

Rotação de culturas II

Rotação de culturas III

Floresta subtropical

Rotação de culturas I

Rotação de culturas II

Rotação de culturas III

Floresta subtropical

Rotação de culturas I

Rotação de culturas II

Rotação de culturas III

Floresta subtropical

\begin{tabular}{cccc}
\hline \multicolumn{3}{c}{$0-0,5 \mathrm{~m}$} & \multicolumn{2}{c}{$0,10-0,15 \mathrm{~m}$} \\
\hline 2001 & 2005 & 2001 & 2005 \\
\hline & Densidade do solo $\left(\mathrm{Mg} \mathrm{m}^{-3}\right)$ \\
$1,30 \mathrm{~b}$ & $1,05 \mathrm{~A}$ & $1,41 \mathrm{~b}$ & $1,34 \mathrm{~A}$ \\
$1,27 \mathrm{~b}$ & $1,10 \mathrm{~A}$ & $1,34 \mathrm{~b}$ & $1,25 \mathrm{~A}$ \\
$1,25 \mathrm{~b}$ & $1,06 \mathrm{a}$ & $1,35 \mathrm{~b}$ & $1,23 \mathrm{a}$ \\
$1,26 \mathrm{~b}$ & $1,03 \mathrm{a}$ & $1,35 \mathrm{~b}$ & $1,19 \mathrm{a}$ \\
$0,88 \mathrm{a}$ & $0,88 \mathrm{a}$ & $1,02 \mathrm{a}$ & $1,02 \mathrm{a}$
\end{tabular}

Porosidade total $\left(\mathrm{m}^{3} \mathrm{~m}^{-3}\right)$

$0,501 \mathrm{~b}$

$0,514 \mathrm{~b}$

$0,521 \mathrm{~b}$

$0,519 \mathrm{~b}$

0,670 a

0,411 a

0,374 a

0,375 a

$0,392 \mathrm{a}$

0,337 a

$0,090 \mathrm{~b}$

$0,144 \mathrm{~b}$

$0,145 \mathrm{~b}$

$0,126 \mathrm{~b}$

0,334 a

$1,30 \mathrm{~b}$

$1,25 \mathrm{~b}$

$1,27 \mathrm{~b}$

0,88 a

$0,502 \mathrm{~b}$

$0,522 \mathrm{~b}$

$0,512 \mathrm{~b}$

0,670 a

$0,392 \mathrm{a}$

0,386 a

0,388 a

$0,337 \mathrm{a}$

$0,115 \mathrm{~b}$

$0,136 \mathrm{~b}$

$0,124 \mathrm{~b}$

0,334 a

$\begin{array}{ll}0,560 \mathrm{a} & 0,462 \mathrm{a} \\ 0,550 \mathrm{a} & 0,489 \mathrm{a} \\ 0,560 \mathrm{a} & 0,485 \mathrm{a} \\ 0,570 \mathrm{a} & 0,485 \mathrm{a} \\ 0,670 \mathrm{a} & 0,621 \mathrm{a}\end{array}$

0,460 a

0,490 a

0,490 a

0,500 a

0,620 a

Microporosidade $\left(\mathrm{m}^{3} \mathrm{~m}^{-3}\right)$

$\begin{array}{lll}0,370 \mathrm{~b} & 0,400 \mathrm{a} & 0,370 \mathrm{~b} \\ 0,330 \mathrm{~b} & 0,400 \mathrm{a} & 0,360 \mathrm{~b} \\ 0,330 \mathrm{~b} & 0,406 \mathrm{a} & 0,370 \mathrm{~b} \\ 0,330 \mathrm{~b} & 0,395 \mathrm{a} & 0,350 \mathrm{~b} \\ 0,340 \mathrm{a} & 0,332 \mathrm{a} & 0,330 \mathrm{a}\end{array}$

Macroporosidade $\left(\mathrm{m}^{3} \mathrm{~m}^{-3}\right)$

$\begin{array}{lll}0,190 \mathrm{a} & 0,071 \mathrm{a} & 0,090 \mathrm{a} \\ 0,220 \mathrm{a} & 0,088 \mathrm{~b} & 0,130 \mathrm{a} \\ 0,230 \mathrm{a} & 0,079 \mathrm{~b} & 0,130 \mathrm{a} \\ 0,240 \mathrm{a} & 0,089 \mathrm{~b} & 0,150 \mathrm{a} \\ 0,330 \mathrm{a} & 0,288 \mathrm{a} & 0,290 \mathrm{a}\end{array}$

Densidade do solo $\left(\mathrm{Mg} \mathrm{m}^{-3}\right)$

$\begin{array}{lll}1,06 \mathrm{a} & 1,38 \mathrm{a} & 1,30 \mathrm{a} \\ 1,08 \mathrm{a} & 1,35 \mathrm{~b} & 1,23 \mathrm{a} \\ 1,05 \mathrm{a} & 1,36 \mathrm{~b} & 1,25 \mathrm{a} \\ 0,88 \mathrm{a} & 1,02 \mathrm{a} & 1,02 \mathrm{a}\end{array}$

Porosidade total $\left(\mathrm{m}^{3} \mathrm{~m}^{-3}\right)$

$\begin{array}{lll}0,570 \mathrm{a} & 0,494 \mathrm{a} & 0,480 \mathrm{a} \\ 0,560 \mathrm{a} & 0,485 \mathrm{a} & 0,490 \mathrm{a} \\ 0,560 \mathrm{a} & 0,478 \mathrm{a} & 0,490 \mathrm{a} \\ 0,670 \mathrm{a} & 0,621 \mathrm{a} & 0,620 \mathrm{a}\end{array}$

Microporosidade $\left(\mathrm{m}^{3} \mathrm{~m}^{-3}\right)$

$\begin{array}{lll}0,350 \mathrm{~b} & 0,405 \mathrm{a} & 0,370 \mathrm{~b} \\ 0,350 \mathrm{~b} & 0,401 \mathrm{a} & 0,350 \mathrm{~b} \\ 0,340 \mathrm{~b} & 0,398 \mathrm{a} & 0,360 \mathrm{~b} \\ 0,340 \mathrm{a} & 0,332 \mathrm{a} & 0,330 \mathrm{a}\end{array}$

Macroporosidade $\left(\mathrm{m}^{3} \mathrm{~m}^{-3}\right)$

$\begin{array}{lll}0,230 \mathrm{a} & 0,069 \mathrm{~b} & 0,110 \mathrm{a} \\ 0,210 \mathrm{a} & 0,089 \mathrm{~b} & 0,130 \mathrm{a} \\ 0,220 \mathrm{a} & 0,082 \mathrm{~b} & 0,120 \mathrm{a} \\ 0,330 \mathrm{a} & 0,288 \mathrm{a} & 0,290 \mathrm{a}\end{array}$

Sistemas de rotação de culturas: I: trigo/soja; sucessão II: trigo/soja e ervilhaca/milho; sucessão III: trigo/soja, ervilhaca/milho e aveia branca/soja. Médias seguidas da mesma letra por profundidade, em manejo de solo e rotação de culturas, na horizontal, não apresentam diferenças, pelo teste de Duncan, a 5\% de probabilidade de erro. 
Tabela 2. Valores de densidade de solo e de porosidade total, nas camadas de solo de 0-0,5 e 0,10-0,15 m, determinados após as culturas de inverno, em quatro sistemas de manejo de solo e em floresta subtropical, em 2001

\begin{tabular}{|c|c|c|c|}
\hline \multirow{2}{*}{ Sistema de produção } & \multicolumn{3}{|c|}{ Profundidade $(\mathrm{m})$} \\
\hline & $0-0,5$ & $0,10-0,15$ & $0-0,5 \times 0,10-0,15$ \\
\hline & Densidade de solo $\left(\mathrm{Mg} \mathrm{m}^{-3}\right)$ & & Contraste entre profundidades $(\mathrm{P}>\mathrm{F})$ \\
\hline PD & 1,20 & 1,38 & $* *$ \\
\hline PCD & 1,26 & 1,38 & $* *$ \\
\hline PCA & 1,19 & 1,37 & $* *$ \\
\hline CM & 1,25 & 1,38 & $* *$ \\
\hline FST & 0,89 & 1,24 & * \\
\hline
\end{tabular}

\section{PD $\times$ PCD \\ $P D \times P C A$ \\ $\mathrm{PD} \times \mathrm{CM}$ \\ $P D \times F S T$ \\ PCD $\times$ PCA \\ $P C D \times C M$ \\ PCD $\times$ FST \\ PCA $\times$ CM \\ PCA $\times$ FST \\ CM $\times$ FST}

PD
PCD
PCA
CM
FST
PD $\times$ PCD
PD $\times$ PCA
PD $\times$ CM
PD $\times$ FST
PCD $\times$ PCA
PCD $\times$ CM
PCD $\times$ FST
PCA $\times$ CM
PCA $\times$ FST
CM $\times$ FST

Contraste entre sistemas

$\begin{array}{ll}\text { ns } & \text { ns } \\ \text { ns } & \text { ns } \\ \text { ns } & \text { ns } \\ * * & * * \\ * & \text { ns } \\ \text { ns } & \text { ns } \\ * * & * * \\ \text { ns } & \text { ns } \\ * * & * * \\ * * & * *\end{array}$

Porosidade total $\left(\mathrm{m}^{3} \mathrm{~m}^{-3}\right)$

$\begin{array}{ll}0,560 & 0,440 \\ 0,520 & 0,450 \\ 0,530 & 0,460 \\ 0,550 & 0,440 \\ 0,670 & 0,520\end{array}$

Contraste entre sistemas

$\begin{array}{ll}* * & \mathrm{~ns} \\ * & \mathrm{~ns} \\ \mathrm{~ns} & \mathrm{~ns} \\ * * & * * \\ \mathrm{~ns} & \mathrm{~ns} \\ \mathrm{~ns} & \mathrm{~ns} \\ * * & * * \\ \mathrm{~ns} & \mathrm{~ns} \\ * * & * * \\ * * & * *\end{array}$

ns = não significativo; = nível de significância de 5\%; ** = nível de significância de $1 \%$.

PD: plantio direto; PCD: preparo convencional de solo com arado de discos; PCA: preparo de solo com arado de aivecas; CM: cultivo mínimo; FST: floresta subtropical.

Como a densidade de solo é uma característica considerada na avaliação do estado estrutural do solo devido à correlação desse atributo com a compactação de solo, esta, nos sistemas de manejo, foi elevada, embora o valor, na camada de 0 a 0,05 $\mathrm{m}$, manteve-se abaixo de um valor considerado como limitante para os Latossolos argilosos do Rio Grande do Sul. De acordo com Klein e Libardi (2000) e CÂMARA e KLEIN (2007), esse valor situa-se ao redor de 1,40 $\mathrm{Mg} \mathrm{m}^{-3}$, o qual nos solos da região, representa intervalo hídrico ótimo crítico, isto é, a faixa de umidade e aeração do solo não limitante, para resistência à penetração está entre 2 e $3 \mathrm{MPa}$.

DERPSCH et al. (1986) observaram, em PD, maior densidade de solo na camada superficial, decrescendo com a profundidade. Neste estudo, os elevados valores de densidade de solo verificados na camada de 0,10$0,15 \mathrm{~m}$, em relação à superficial podem ser, ao menos em parte, atribuídos à presença de camada compactada residual resultante de operações de preparo de solo com aração e gradagem anteriores ao início do experimento, denominada pé-de-grade. 
Tabela 3. Valores de densidade de solo e de porosidade total, nas camadas de solo de 0-0,5 e 0,10-0,15 m, determinados após as culturas de inverno, em quatro sistemas de manejo de solo e em floresta subtropical, em 2005

\begin{tabular}{|c|c|c|c|}
\hline \multirow{2}{*}{ Sistema de manejo } & \multicolumn{3}{|c|}{ Camada (m) } \\
\hline & $0-0,5$ & $0,10-0,15$ & $0-0,5 \times 0,10-0,15$ \\
\hline & Densidade de solo $\left(\mathrm{Mg} \mathrm{m}^{-3}\right)$ & & Contraste entre camadas $(\mathrm{P}>\mathrm{F})$ \\
\hline PD & 1,05 & 1,34 & $* *$ \\
\hline PCD & 1,10 & 1,25 & $* *$ \\
\hline PCA & 1,06 & 1,23 & $* *$ \\
\hline $\mathrm{CM}$ & 1,03 & 1,19 & $* *$ \\
\hline \multirow[t]{2}{*}{ FST } & 0,88 & 1,02 & ns \\
\hline & Contraste entre sistemas & & \\
\hline PD $\times$ PCD & ns & $* *$ & \\
\hline PD $\times$ PCA & ns & $* *$ & \\
\hline $\mathrm{PD} \times \mathrm{CM}$ & ns & $* *$ & \\
\hline PD $\times$ FST & $* *$ & $* *$ & \\
\hline PCD $\times$ PCA & ns & ns & \\
\hline $\mathrm{PCD} \times \mathrm{CM}$ & * & ns & \\
\hline PCD $\times$ FST & $* *$ & $* *$ & \\
\hline PCA $\times$ CM & ns & ns & \\
\hline PCA $\times$ FST & $* *$ & $* *$ & \\
\hline \multirow[t]{2}{*}{$\mathrm{CM} \times \mathrm{FST}$} & $* *$ & $* *$ & \\
\hline & Porosidade total $\left(\mathrm{m}^{3} \mathrm{~m}^{-3}\right)$ & & Contraste entre camadas $(P>F)$ \\
\hline PD & 0,560 & 0,460 & $* *$ \\
\hline PCD & 0,550 & 0,490 & $* *$ \\
\hline PCA & 0,560 & 0,490 & $* *$ \\
\hline $\mathrm{CM}$ & 0,570 & 0,500 & $* *$ \\
\hline \multirow[t]{2}{*}{ FST } & 0,670 & 0,620 & ns \\
\hline & Contraste entre sistemas & & \\
\hline$P D \times P C D$ & ns & $* *$ & \\
\hline PD $\times$ PCA & ns & $* *$ & \\
\hline $\mathrm{PD} \times \mathrm{CM}$ & ns & $* *$ & \\
\hline $\mathrm{PD} \times \mathrm{FST}$ & $* *$ & $* *$ & \\
\hline PCD $\times$ PCA & ns & ns & \\
\hline $\mathrm{PCD} \times \mathrm{CM}$ & * & ns & \\
\hline PCD $\times$ FST & $* *$ & $* *$ & \\
\hline PCA $\times$ CM & ns & ns & \\
\hline PCA $\times$ FST & $* *$ & $* *$ & \\
\hline CM $\times$ FST & $* *$ & $* *$ & \\
\hline
\end{tabular}

ns = não significativo; = nível de significância de $5 \% ;{ }^{* *}=$ nível de significância de $1 \%$.

PD: plantio direto; PCD: preparo convencional de solo com arado de discos; PCA: preparo de solo com arado de aivecas; CM: cultivo mínimo; FST: floresta subtropical.

Na camada superficial, os valores da porosidade total (Tabela 3), em todos os SMSs, em 2005, foram mais elevados que aqueles de 2001 (Tabela 2), após quatro anos de cultivo. Houve diferenças quanto à porosidade total, entre os SMSs estudados, na camada de 0,10 a $0,15 \mathrm{~m}$, onde o PD proporcionou menor porosidade total, em comparação aos demais SMSs. A porosidade total foi influenciada pelo SMSs, em decorrência das alterações verificadas na densidade do solo. Assim, sob PD, verificou-se na camada de 0,10 a 0,15 m menor volume de macroporos, com consequente incremento no volume de microporos
(Tabela 4), podendo resultar em redução da taxa de infiltração de água neste sistema de manejo de solo, em relação aos demais SMSs, conforme fora observado em alguns trabalhos (SECCo et al., 2005; Beutler et al., 2001). Como é previsível, na FST ocorreu maior porosidade total, em relação aos SMSs, em ambas as camadas estudadas, permitindo constatar que a porosidade também é afetada pelo uso agrícola. Observações semelhantes foram reportadas por ALBUQUERQUE et al. (2001), que constataram na FST porosidade total bem maior que as de solos sob PD e PCD, nas camadas de 0 a $0,05 \mathrm{~m}$ e de 0,10 a $0,15 \mathrm{~m}$. 
Tabela 4. Valores de microporosidade e de macroporosidade, nas camadas de solo de 0-0,5 e 0,10-0,15 m, determinados após as culturas de verão, em quatro sistemas de manejo de solo e em floresta subtropical, em 2001

\begin{tabular}{|c|c|c|c|}
\hline \multirow{2}{*}{ Sistema de produção } & \multicolumn{3}{|c|}{ Profundidade $(\mathrm{m})$} \\
\hline & $0-0,5$ & $0,10-0,15$ & $0-05 \times 0,10-0,15$ \\
\hline & Microporosidade $\left(\mathrm{m}^{3} \mathrm{~m}^{-3}\right)$ & & Contraste entre profundidades $(\mathrm{P}>\mathrm{F})$ \\
\hline PD & 0,430 & 0,370 & $* *$ \\
\hline PCD & 0,380 & 0,400 & ns \\
\hline PCA & 0,370 & 0,410 & $* *$ \\
\hline $\mathrm{CM}$ & 0,410 & 0,380 & ** \\
\hline FST & 0,440 & 0,340 & ns \\
\hline
\end{tabular}

$P D \times P C D$

$P D \times P C A$

$\mathrm{PD} \times \mathrm{CM}$

$P D \times$ FST

PCD $\times$ PCA

$P C D \times C M$

PCD $\times$ FST

PCA $\times$ CM

PCA $\times$ FST

CM $\times$ FST

PD

PCD

PCA

$\mathrm{CM}$

FST

PD $\times$ PCD

PD $\times$ PCA

$\mathrm{PD} \times \mathrm{CM}$

$P D \times$ FST

PCD $\times$ PCA

$P C D \times C M$

PCD $\times$ FST

PCA $\times$ CM

PCA $\times$ FST

CM $\times$ FST

PD

PCD

PCA

CM

FST

$P D \times P C D$

$P D \times P C A$

$\mathrm{PD} \times \mathrm{CM}$

$P D \times$ FST

$P C D \times P C A$

PCD $\times$ CM

PCD $\times$ FST

PCA $\times$ CM

CM $\times$ FST
PCA $\times$ FST

Contraste entre sistemas

$* *$
$* *$
$\mathrm{~ns}$
$\mathrm{~ns}$
$\mathrm{~ns}$
$* *$
$*$
$* *$
$* *$
$\mathrm{~ns}$

Macroporosidade $\left(\mathrm{m}^{3} \mathrm{~m}^{-3}\right)$

$\begin{array}{ll}0,130 & 0,070 \\ 0,140 & 0,050 \\ 0,160 & 0,050 \\ 0,130 & 0,060 \\ 0,230 & 0,180\end{array}$

Contraste entre sistemas

$\begin{array}{cc}\text { ns } & * \\ \text { ns } & * * \\ \text { ns } & \text { ns } \\ * * & * * \\ \text { ns } & \text { ns } \\ \text { ns } & \text { ns } \\ * & * * \\ \text { ns } & \text { ns } \\ * & * * \\ * * & * *\end{array}$

Resistência à penetração $\left(\mathrm{kgf} \mathrm{cm}^{-2}\right)$

2,14

1,91

2,04

1,99

1,20

Contraste entre sistemas

ns = não significativo; = nível de significância de 5\%; ** = nível de significância de $1 \%$.

PD: plantio direto; PCD: preparo convencional de solo com arado de discos; PCA: preparo de solo com arado de aivecas; CM: cultivo mínimo; FST: floresta subtropical.
Contraste entre profundidades $(\mathrm{P}>\mathrm{F})$

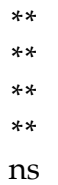

Contraste entre profundidades $(\mathrm{P}>\mathrm{F})$

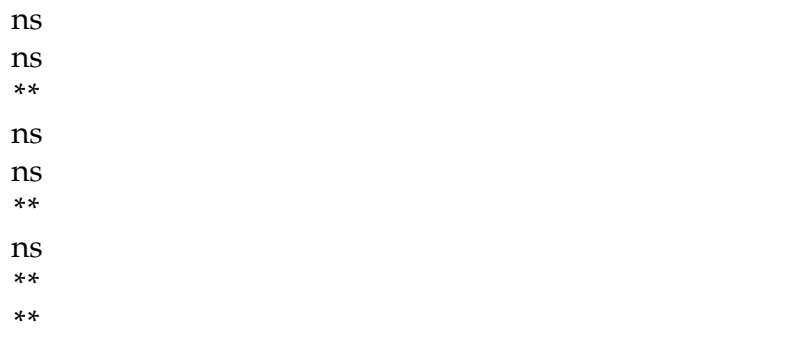


Tabela 5. Valores de microporosidade e de macroporosidade, nas camadas de solo de 0-0,5 e 0,10-0,15 m, determinados após as culturas de verão, em quatro sistemas de manejo de solo e em floresta subtropical, em 2005

\begin{tabular}{|c|c|c|c|}
\hline \multirow{2}{*}{ Sistema de produção } & \multicolumn{3}{|c|}{ Camada (m) } \\
\hline & $0-0,5$ & $0,10-0,15$ & $0-0,5 \times 0,10-0,15$ \\
\hline & Microporosidade $\left(\mathrm{m}^{3} \mathrm{~m}^{-3}\right)$ & & Contraste entre camadas $(\mathrm{P}>\mathrm{F})$ \\
\hline PD & 0,370 & 0,370 & ns \\
\hline PCD & 0,330 & 0,360 & $* *$ \\
\hline PCA & 0,330 & 0,370 & $* *$ \\
\hline CM & 0,330 & 0,350 & ns \\
\hline FST & 0,340 & 0,330 & ns \\
\hline
\end{tabular}

\section{PD $\times$ PCD \\ $P D \times P C A$ \\ $\mathrm{PD} \times \mathrm{CM}$ \\ $P D \times F S T$ \\ PCD $\times$ PCA \\ $P C D \times C M$ \\ PCD $\times$ FST \\ PCA $\times$ CM \\ PCA $\times$ FST \\ CM x FST}

PD

PCD

$\mathrm{CM}$

FST

PD $\times$ PCD

$P D \times P C A$

$\mathrm{PD} \times \mathrm{CM}$

PD $\times$ FST

PCD $\times$ PCA

PCD $\times$ CM

PCD $\times$ FST

PCA $\times$ CM

PCA $\times$ FST

CM $\times$ FST

Contraste entre sistemas

$\begin{array}{ll}* * & \mathrm{~ns} \\ * * & \mathrm{~ns} \\ * * & * * \\ * & * * \\ \mathrm{~ns} & \mathrm{~ns} \\ \mathrm{~ns} & * * \\ \mathrm{~ns} & * * \\ \mathrm{~ns} & * * \\ \mathrm{~ns} & * * \\ \mathrm{~ns} & \mathrm{~ns}\end{array}$

$$
\text { Macroporosidade }\left(\mathrm{m}^{3} \mathrm{~m}^{-3}\right)
$$

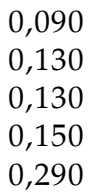

Contraste entre camadas $(\mathrm{P}>\mathrm{F})$

$\begin{array}{ll}0,190 & 0,090 \\ 0,220 & 0,130 \\ 0,230 & 0,130 \\ 0,240 & 0,150 \\ 0,330 & 0,290\end{array}$

$* *$
$* *$
$* *$
$\mathrm{~ns}$

Contraste entre sistemas

$\begin{array}{ll}* * & * * \\ * * & * * \\ * * & * * \\ * * & * * \\ \mathrm{~ns} & \mathrm{~ns} \\ \mathrm{~ns} & \mathrm{~ns} \\ * * & * * \\ \mathrm{~ns} & \mathrm{~ns} \\ * * & * * \\ * * & * *\end{array}$

ns = não significativo; = nível de significância de 5\%; ** = nível de significância de $1 \%$.

PD: plantio direto; PCD: preparo convencional de solo com arado de discos; PCA: preparo de solo com arado de aivecas; CM: cultivo mínimo; FST: floresta subtropical.

Porém, Araújo et al. (2004), avaliando diferentes cultivos sob PCD alternados com CM, observaram maiores valores de porosidade total nestes sistemas do que sob FST. DA Ros et al. (1997) constataram, em solos sob preparo convencional com aração e gradagem, porosidade total em camadas superficial e subsuperficial, maior que a do PD.

Para porosidade total, houve diferença entre as duas profundidades de amostragem em todos os SMSs estudados e na FST (Tabelas 1, 2 e 3) seguindo a mesma tendência inversa da densidade do solo, ainda que, neste estudo, a porosidade total não foi obtida pela relação com densidade. A porosidade total foi maior na camada de 0 a $0,05 \mathrm{~m}$ do que na camada de 0,10 a $0,15 \mathrm{~m}$, principalmente em razão da deposição de resíduos culturais na superfície, o que aumentou o nível de matéria orgânica em todos SMSs (SANTos e Tomm, 2003); o efeito do sulcador da semeadora, porém, atuando nesta camada a cada $0,17 \mathrm{~m}$ para os cereais de inverno, pode ter contribuído bastante para a melhoria, não só da densidade, como da porosidade na camada superficial (CÂMARA e KLEIN, 2005). Resultados equivalentes foram observados, para porosidade total, por AlbuQuerQue et al. (2001), apenas no PCD, para as mesmas camadas estudadas. 
Os valores de microporosidade, em 2005 (Tabela 5), em todos os SMSs, nas duas camadas estudadas foram menores, em relação ao observado, em 2001 (Tabela 4). Secco et al. (2005), somente para PD, observaram o mesmo, na camada de 0 a 0,07 m em três anos de estudo. Houve diferenças entre as médias de microporosidade em todos os SMSs, principalmente na camada de 0 a $0,05 \mathrm{~m}$. Sob PD o valor para microporosidade foi maior do que nos demais SMSs, na camada de 0 a $0,05 \mathrm{~m}$ e do PD para o CM, na camada de 0,10 a 0,15 m. Nessa mesma camada, sob PCA houve maior microporosidade que sob PCD e CM. Stone e Silveira (2001) e Bertol et al. (2004) verificaram microporosidade maior sob PD, em comparação aos preparos convencionais de solo, na camada de 0 a $0,10 \mathrm{~m}$. Na FST, notou-se menor valor de microporosidade, em comparação ao PD, na camada superficial e em todos os SMSs, na segunda camada estudada. O aumento da microporosidade pode ser considerado um reflexo da redução da estruturação e atribuído à redução no volume de macroporos, o que torna prejudicial ao desenvolvimento das plantas (KLeIN e LibARDI, 2000). Albuguerque et al. (2001) constatou na FST valor de microporosidade maior do que o do PCD, na camada de 0 a $0,05 \mathrm{~m}$. De acordo com os mesmos autores, nessa mesma camada, o valor de microporosidade no PD ficou em posição intermediária, em relação aos SMSs estudados. Oliveira et al. (2004), estudando um Latossolo Vermelho Distrófico típico, em Planaltina (DF), verificaram, na camada de 0 a $0,05 \mathrm{~m}$, maior valor de microporosidade sob $\mathrm{PD}$, em relação ao do PCD e ao do Cerrado nativo. De acordo com esses mesmos autores, sob PD a disponibilidade de água foi maior do que sob PCD e Cerrado nativo, na camada de 0 a $0,05 \mathrm{~m}$. Em Latossolos sob vegetação natural, a microporosidade geralmente é menor que nos mesmos solos sob uso agrícola devido ao maior diâmetro de agregação (SÁ et al., 2000).

Em 2001 e 2005, houve diferenças entre as profundidades de solo, para microporosidade, nos tratamentos estudados onde houve revolvimento (PDA e PDC) ou mobilização (CM) (Tabelas 1, 4 e 5). Essa diferença pode ser decorrente do efeito da movimentação do solo com arado ou escarificador, que determina uma reorganização estrutural do solo diferente da situação em que não ocorre revolvimento. Porém, ANDreola et al. (2000) e Costa et al. (2003), estudando SMSs, não verificaram diferenças nos valores de microporosidade entre as distintas camadas de solo.

Os valores de macroporosidade (Tabela 5), em 2005, em todos os SMSs e nas camadas estudadas, aumentaram após quatro anos de cultivo (Tabela 4). Resultado semelhante foi observado por SECCO et al.
(2005), na camada de 0 a $0,07 \mathrm{~m}$, em três anos de estudo, para PD, CM e PCD. O valor de macroporosidade foi mais elevado no PCD, PCA e $\mathrm{CM}$, em comparação ao $\mathrm{PD}$, nas duas camadas estudadas. O menor volume de macroporos, com consequente maior volume de microporos na superfície do solo, no PD, pode reduzir a taxa de infiltração de água neste sistema de manejo, em relação ao preparo convencional de solo (BERTOL et al., 2004). De acordo com os mesmos autores, esse problema torna-se crítico, com volume de macroporos menor do que $0,10 \mathrm{~m}^{3} \mathrm{~m}^{-3}$. Por sua vez, na FST, houve maior macroporosidade do que nos SMSs, em ambas as camadas estudadas (Tabelas 4 e 5). Como era de se esperar, a floresta subtropical, em razão das condições naturais favoráveis à agregação de solo, possui volume de macroporos maior do que o dos SMSs estudados. Em estudo realizado por AlbuQuerque et al. (2001), observou-se no solo da floresta subtropical valor de macroporosidade maior do que em PD, nas camadas de 0 a $0,05 \mathrm{~m}$ e de 0,10 a $0,15 \mathrm{~m}$ respectivamente. Stone e Silveira (2001) verificaram, em condições de Cerrado, que sob PCD, preparo somente com arado e grade, os maiores valores de macroporosidade, em relação ao PD, ocorreram na camada de 0 a $0,10 \mathrm{~m}$. Oliveira et al. (2004) também verificaram menor valor de macroporosidade sob PD, em comparação com o PCD e com o Cerrado nativo, na camada de 0 a $0,05 \mathrm{~m}$.

Houve diferenças entre as profundidades, nos SMSs, para a macroporosidade (Tabelas 4 e 5). A macroporosidade do solo na camada de 0 a $0,5 \mathrm{~m}$ foi maior que na camada de 0,10 a 0,15 m. A redução da macroporosidade, na camada logo abaixo da superficial, pode ser consequência de menor nível de matéria orgânica em subsuperfície. TREIN et al. (1991) verificaram em $\mathrm{PD}$, maior macroporosidade na camada de 0 a 0,08 $\mathrm{m}$ em relação à camada de 0,08 a $0,15 \mathrm{~m}$, porém, na floresta subtropical, além de contar com maiores valores não se verificou diferença entre as profundidades estudadas para macroporosidade indicando que o uso do solo altera desfavoravelmente este atributo. Maiores valores de macroporosidade nas camadas superficiais refletem efeito positivo da matéria orgânica na estruturação de solos (DEXTER, 1991).

Rotação de culturas - Os sistemas de rotação de culturas (SRCs) não proporcionaram diferenças para densidade de solo (Tabelas 1 e 6). A densidade de solo, em todas as rotações e sequência trigo/soja, em 2005, revelou redução nos valores, em comparação ao verificado em 2001, nas camadas de 0 a $0,05 \mathrm{~m}$ e de 0,10 a $0,15 \mathrm{~m}$, após quatro anos de cultivo (Tabela 7). 
Tabela 6. Valores de densidade de solo e de porosidade total, nas camadas de solo de 0-0,5 e 0,10-0,15 m, determinados após as culturas de inverno, em três rotações de culturas e em floresta subtropical, em 2001

\begin{tabular}{|c|c|c|c|}
\hline \multirow{2}{*}{ Sistema de produção } & \multicolumn{3}{|c|}{ Profundidade $(\mathrm{m})$} \\
\hline & $0-0,5$ & $0,10-0,15$ & $0-0,5 \times 0,10-0,15$ \\
\hline \multicolumn{3}{|c|}{ Densidade de solo $\left(\mathrm{Mg} \mathrm{m}^{-3}\right)$} & Contraste entre profundidades $(\mathrm{P}>\mathrm{F})$ \\
\hline Rotação I & 1,22 & 1,39 & ** \\
\hline Rotação II & 1,22 & 1,36 & $* *$ \\
\hline Rotação III & 1,23 & 1,39 & $* *$ \\
\hline FST & 0,89 & 1,24 & * \\
\hline \multicolumn{4}{|c|}{ Contraste entre sistemas } \\
\hline I $x$ II & ns & ns & \\
\hline I x III & ns & ns & \\
\hline I x FST & $* *$ & ** & \\
\hline II x III & ns & ns & \\
\hline II x FST & $* *$ & ** & \\
\hline III $x$ FST & $* *$ & ** & \\
\hline & Porosidade total $\left(\mathrm{m}^{3} \mathrm{~m}^{-3}\right)$ & & Contraste entre profundidades $(\mathrm{P}>\mathrm{F})$ \\
\hline Rotação I & 0,550 & 0,440 & $* *$ \\
\hline Rotação II & 0,540 & 0,450 & $* *$ \\
\hline Rotação III & 0,540 & 0,450 & $* *$ \\
\hline FST & 0,670 & 0,520 & * \\
\hline \multicolumn{4}{|c|}{ Contraste entre sistemas } \\
\hline I x II & ns & ns & \\
\hline I x III & ns & ns & \\
\hline $\mathrm{I} \times \mathrm{FST}$ & $* *$ & $* *$ & \\
\hline II x III & ns & ns & \\
\hline II $x$ FST & $* *$ & $* *$ & \\
\hline III $x$ FST & $* *$ & $* *$ & \\
\hline
\end{tabular}

ns = não significativo; = nível de significância de 5\%; ** = nível de significância de $1 \%$.

Sistema I: trigo/soja; sistema II: trigo/soja e ervilhaca/milho; sistema III: trigo/soja, ervilhaca/milho e aveia branca/soja; FST: floresta subtropical.

Esses resultados são equiparáveis aos verificados por AlbuQUERQUe et al. (1995), COSTA et al. (2003) e BERTOL et al. (2004), os quais atribuíram a diminuição da densidade de solo à melhoria na qualidade física do solo decorrente possivelmente do acúmulo de matéria orgânica, da atividade da fauna edáfica e de raízes, as quais atuam na formação de canais (ou bioporos). De acordo com BerTol et al. (2004), o efeito da rotação ou sucessão de culturas tem sido mais demorado do que os dos SMSs, em modificar a densidade do solo. Nas duas camadas estudadas, o solo da FST, que preserva a condição estrutural original, foi o de menor densidade, em comparação aos SRCs, indicando que estes sistemas de rotação não restauram os atributos físicos aos níveis comparáveis com o solo em condição natural.

Os resultados da avaliação dos atributos físicos verificados nos SRCs permitem afirmar que não houve ocorrência de severa compactação de solo, embora os valores observados na subsuperfície situam-se próximos do valor considerado, por KLEIN e CÂmara (2007), como crítico para latossolos argilosos, quando atingem resistência à penetração entre 2 e $3 \mathrm{MPa}$, a $75 \%$ da capacidade de campo, uma condição restritiva frequente nos solos da região durante o período das safras de verão (MARCOLIN, 2006). A densidade de solo foi menor na camada superficial em relação à camada subsuperficial. SPERA et al. $(2004 ; 2006)$ observaram, em modelos de produção integrada com pastagens anuais de inverno ou perenes, no mesmo tipo de solo, menor valor de densidade de solo na camada de 0 a $0,05 \mathrm{~m}$, em relação à camada de 0,10 a 0,15 m.

Nas duas camadas estudadas, o valor da porosidade total (Tabela 7), em todos os SRCs verificado em 2005 foi menor que o observado em 2001 (Tabela 6). Maior valor para porosidade total na camada superficial reflete menor densidade do solo e pode ser atribuída ao acúmulo de material orgânico nessa camada, principalmente, considerando o nãorevolvimento de solo. A matéria orgânica gerada pelos resíduos mantidos em superfície pode ter reestruturado os agregados do solo. Também, não houve diferença entre os SRCs. SPERA et al. (2006), trabalhando com 
modelos de produção integrados com pastagens anuais de inverno e de verão, obtiveram resultados equivalentes para os valores de porosidade total. Todavia, no solo de FST constatou-se maior valor de porosidade total do que nos dos SRCs, refletindo as condições naturais de estruturação dos latossolos. Nesse caso, em relação à FST, constataram-se menores valores da macroporosidade em todos os SRCs estudados, com conseqüente redução na porosidade total, resultante do uso agrícola. Verificaram-se diferenças na porosidade total entre as profundidades de todos os SRCs. A porosidade total foi maior na camada superficial em comparação à camada subsuperficial, indicando degradação estrutural restrita a esta camada, o que se evidenciou com a redução da macroporosidade. Resultados semelhantes foram observados por SPERA et al. (2004; 2006), com modelos de produção integrados com pastagens anuais e perenes.

Os valores de microporosidade em 2005 (Tabela 9), em todos os SRCs, em ambas as camadas estudadas foi menor, em relação ao observado em 2001 (Tabela 8). Os volumes de microporos, macroporos e de total de poros não foram influenciados pelos SRCs. Também não se constataram interações entre os tratamentos tipos de manejo de solo e sistemas de rotação de culturas, evidenciando que os SRCs não promoveram alterações adicionais nos atributos de solo estudados. AlbuQuerque et al. (1995), trabalhando com seqüência trigo/soja e rotações de culturas, igualmente não obtiveram diferenças entre as médias da microporosidade na camada de 0,01 a $0,09 \mathrm{~m}$. Nessa mesma camada, no solo da FST os valores de microporosidade foram iguais ou menores, em relação aos SRCs, decorrentes da maior proporção de macroporos sustentados pela maior agregação nos solos da FST. Nas sucessões trigo/soja, e trigo/soja, ervilhaca/milho e aveia branca/soja foram verificadas diferenças para microporosidade entre as profundidades estudadas. Nesse caso, a microporosidade foi maior na camada de 0 a $0,05 \mathrm{~m}$ do que na camada de 0,10 a $0,15 \mathrm{~m}$.

Tabela 7. Valores de densidade do solo e de porosidade total, nas camadas de solo de 0-0,5 e 0,10-0,15 m, determinados após as culturas de verão, em três rotações de culturas e em floresta subtropical, em 2005

\begin{tabular}{|c|c|c|c|}
\hline \multirow{2}{*}{$\begin{array}{l}\text { Sistema de rotação } \\
\text { de culturas }\end{array}$} & \multicolumn{3}{|c|}{ Camada (m) } \\
\hline & $0-0,5$ & $0,10-0,15$ & $0-0,5 \times 0,10-0,15$ \\
\hline & Densidade de solo $\left(\mathrm{Mg} \mathrm{m}^{-3}\right)$ & & Contraste entre camadas $(\mathrm{P}>\mathrm{F})$ \\
\hline Rotação I & 1,06 & 1,30 & $* *$ \\
\hline Rotação II & 1,08 & 1,23 & $* *$ \\
\hline Rotação III & 1,05 & 1,25 & $* *$ \\
\hline \multirow[t]{2}{*}{ FST } & 0,88 & 1,02 & ns \\
\hline & Contraste entre sistemas & & \\
\hline I $x$ II & ns & * & \\
\hline I x III & ns & ns & \\
\hline I x FST & $* *$ & $* *$ & \\
\hline II x III & ns & ns & \\
\hline II $x$ FST & $* *$ & $* *$ & \\
\hline \multirow[t]{2}{*}{ III $\times$ FST } & $* *$ & $* *$ & \\
\hline & Porosidade total $\left(\mathrm{m}^{3} \mathrm{~m}^{-3}\right)$ & & Contraste entre camadas $(\mathrm{P}>\mathrm{F})$ \\
\hline Rotação I & 0,570 & 0,480 & ** \\
\hline Rotação II & 0,560 & 0,490 & $* *$ \\
\hline Rotação III & 0,560 & 0,490 & $* *$ \\
\hline \multirow[t]{2}{*}{ FST } & 0,670 & 0,620 & ns \\
\hline & Contraste entre sistemas & & \\
\hline I x II & ns & ns & \\
\hline I x III & ns & ns & \\
\hline $\mathrm{I} \times \mathrm{FST}$ & $* *$ & $* *$ & \\
\hline II x III & ns & ns & \\
\hline II x FST & $* *$ & $* *$ & \\
\hline III $x$ FST & $* *$ & $* *$ & \\
\hline
\end{tabular}

ns = não significativo; = nível de significância de 5\%; ** = nível de significância de $1 \%$.

Sistemas de rotação de culturas: I: trigo/soja; sucessão II: trigo/soja e ervilhaca/milho; sucessão III: trigo/soja, ervilhaca/milho e aveia branca/soja; FST: floresta subtropical. 
Tabela 8. Valores de microporosidade e de macroporosidade, nas camadas de solo de 0-0,5 e 0,10-0,15 m, determinados após as culturas de verão, em três rotações de culturas e em floresta subtropical, em 2001

\begin{tabular}{|c|c|c|c|}
\hline \multirow{2}{*}{ Sistema de produção } & \multicolumn{3}{|c|}{ Profundidade $(\mathrm{m})$} \\
\hline & $0-05$ & $0,10-0,15$ & $0-0,5 \times 0,10-0,15$ \\
\hline & Microporosidade $\left(\mathrm{m}^{3} \mathrm{~m}^{-3}\right)$ & & Contraste entre profundidades $(\mathrm{P}>\mathrm{F})$ \\
\hline Rotação I & 0,390 & 0,380 & ns \\
\hline Rotação II & 0,390 & 0,390 & ns \\
\hline Rotação III & 0,400 & 0,400 & ns \\
\hline FST & 0,440 & 0,340 & ns \\
\hline
\end{tabular}

Contraste entre sistemas

I $x$ II
I $x$ III
I $x$ FST
II $x$ III
II $x$ FST
III $x$ FST

Rotação I

Rotação II

Rotação III

FST

I $x$ II
I $x$ III
I $x$ FST
II $x$ III
II $x$ FST
III $x$ FST

Rotação I

Rotação II

Rotação III

FST

I x II

I $x$ III

I $x$ FST

II $x$ III

II $x$ FST

III $x$ FST

$\begin{array}{cc}\text { ns } & \text { ns } \\ \text { ns } & \text { ns } \\ \text { ns } & \text { ns } \\ \text { ns } & \text { ns } \\ \text { ns } & * \\ \text { ns } & * *\end{array}$

Macroporosidade $\left(\mathrm{m}^{3} \mathrm{~m}^{-3}\right)$

$\begin{array}{ll}0,150 & 0,060 \\ 0,150 & 0,060 \\ 0,140 & 0,050 \\ 0,230 & 0,180\end{array}$

Contraste entre profundidades $(\mathrm{P}>\mathrm{F})$

0,060

0,050

0,180

Contraste entre sistemas

$\begin{array}{cc}\mathrm{ns} & \mathrm{ns} \\ \mathrm{ns} & \mathrm{ns} \\ * & * * \\ \mathrm{~ns} & \mathrm{~ns} \\ * & * * \\ * * & * *\end{array}$

Resistência à penetração $\left(\mathrm{kgf} \mathrm{cm}^{-2}\right)$

ns

ns

ns

$* *$

**

$* *$

$* *$

ns

S
*
$*$

95
90
2,06
1,20

Contraste entre sistemas
Contraste entre profundidades $(\mathrm{P}>\mathrm{F})$

ns = não significativo; = nível de significância de 5\%; ** = nível de significância de $1 \%$.

Sistema I: trigo/soja; sistema II: trigo/soja e ervilhaca/milho; sistema III: trigo/soja, ervilhaca/milho e aveia branca/soja: FST: floresta subtropical.

Os macroporos estão relacionados com processos vitais para as raízes das plantas, tais como respiração, devendo, portanto, serem manejados visando à sua manutenção e preservação (AITA et al., 2006). Os valores de macroporosidade (Tabela 9), em 2005, em todos os SRCs, em ambas as camadas, aumentaram após quatro anos de cultivo, ao se comparar com a Tabela 8. Não houve diferença entre os SRCs, nas duas camadas estudadas. Resultados equivalentes foram obtidos por Albuquerque et al. (1995) que compararam distintos SRCs com a floresta. A redução da macroporosidade em sistemas de produção agrícola tende a refletir negativamente, diminuindo a porosidade total e aumentando a densidade de solo; no solo da FST constataram-se maiores valores de macroporosidade em comparação aos SRCs, indicando haver maior volume de macroporos em ecossistema naturais (REICHERT et al., 2003). 
Tabela 9. Valores de microporosidade e de macroporosidade, nas camadas de solo de 0-0,5 e 0,10-0,15 m, determinados após as culturas de verão, em três rotações de culturas e em floresta subtropical, em 2005

\begin{tabular}{|c|c|c|c|}
\hline \multirow{2}{*}{$\begin{array}{l}\text { Sistemas de rotação } \\
\text { de culturas }\end{array}$} & \multicolumn{3}{|c|}{ Camada $(\mathrm{m})$} \\
\hline & $0-0,5$ & $0,10-0,15$ & $0-0,5 \times 0,10-0,15$ \\
\hline & Microporosidade $\left(\mathrm{m}^{3} \mathrm{~m}^{-3}\right)$ & & Contraste entre camadas $(P>F)$ \\
\hline Rotação I & 0,350 & 0,370 & * \\
\hline Rotação II & 0,350 & 0,350 & ns \\
\hline Rotação III & 0,340 & 0,360 & $* *$ \\
\hline \multirow[t]{2}{*}{ FST } & 0,340 & 0,330 & ns \\
\hline & Contraste entre sistemas & & \\
\hline I x II & ns & * & \\
\hline I x III & ns & ns & \\
\hline $\mathrm{I} \times \mathrm{FST}$ & ns & $* *$ & \\
\hline II x III & ns & ns & \\
\hline II $x$ FST & ns & $\mathrm{ns}$ & \\
\hline \multirow[t]{2}{*}{ III $x$ FST } & ns & ** & \\
\hline & Macroporosidade $\left(\mathrm{m}^{3} \mathrm{~m}^{-3}\right)$ & & Contraste entre camadas $(\mathrm{P}>\mathrm{F})$ \\
\hline Rotação I & 0,230 & 0,110 & ** \\
\hline Rotação II & 0,210 & 0,130 & ** \\
\hline Rotação III & 0,220 & 0,120 & $* *$ \\
\hline \multirow[t]{2}{*}{ FST } & 0,330 & 0,290 & ns \\
\hline & Contraste entre sistemas & & \\
\hline I x II & ns & ns & \\
\hline I x III & $\mathrm{ns}$ & ns & \\
\hline $\mathrm{I} \times \mathrm{FST}$ & $* *$ & $* *$ & \\
\hline II x III & ns & ns & \\
\hline II x FST & $* *$ & $* *$ & \\
\hline III $x$ FST & $* *$ & $* *$ & \\
\hline
\end{tabular}

ns = não significativo; = nível de significância de 5\%; ** = nível de significância de $1 \%$.

Sistemas de rotação de culturas I: trigo/soja; sucessão II: trigo/soja e ervilhaca/milho; sucessão III: trigo/soja, ervilhaca/milho e aveia branca/soja: FST: floresta subtropical.

\section{CONCLUSÕES}

1. Em um período de quatro anos (2001 a 2005) há alterações nos atributos físicos dos solos entre os distintos tipos de manejo do solo.

2. O sistema conservacionista PD proporciona maior densidade de solo e microporosidade em comparação aos demais, principalmente na camada de 0,10 a $0,15 \mathrm{~m}$ o que confere ao sistema maior intensidade na compactação do solo.

3. Os sistemas de rotação de culturas não afetam os atributos físicos de solo independentemente do tipo de manejo.

\section{REFERÊNCIAS}

AITA, C.; GIACOMINI, S.J. Plantas de cobertura de solo em sistemas agrícolas. In: ALVES, B.J.R.; URQUIAGA, S.; AITA, C.; BODDEY, R.M.; JANTALIA, C.P.; CAMARGO, F.A.O. (Ed.). Manejo de sistemas agrícolas: Impacto no seqüestro de $\mathrm{C}$ e nas emissões de gases de efeito estufa. Porto Alegre: Gênesis, 2006. p.59-80.
ALBUQUERQUE, J.A.; REINERT, D.J.; FIORIN, J.E.; RUEDEL, J.; PETRERE, C.; FONTINELLI, F. Rotação de culturas e sistemas de manejo do solo. Revista Brasileira de Ciência do Solo, v.19, p.115-119, 1995.

ALBUQUERQUE, J.A.; SANGOI, L.; ENDER, M. Efeitos da integração lavoura-pecuária nas propriedades físicas do solo e características da cultura do milho. Revista Brasileira de Ciência do Solo, v.25, p.717-723, 2001.

ANDREOLA, F.; COSTA, L.M.; OLSZEVSKI, N. Influência da cobertura vegetal de inverno e da adubação orgânica e/ou mineral sobre as propriedades físicas de uma Terra Roxa Estruturada. Revista Brasileira de Ciência do Solo, v.24, n.4, p.857-865, 2000.

ANJOS, J.T.; UBERT, A.A.A.; VIZZOTTO, V.J.; LEITE, G.B.; KRIEGER, M. Propriedades físicas em solos sob diferentes sistemas de uso e manejo. Revista Brasileira de Ciência do Solo, v.18, p.139-145, 1994.

ARAUJO, M.A.; TORMENA, C.A.; SILVA, A.P. Propriedades físicas de um Latossolo Vermelho distrófico cultivado e sob mata nativa. Revista Brasileira de Ciência do Solo, v.28, p.337-345, 2004. 
ARSHAD, M.A.; LOWERY, B.; GROSSMANN, B. Physical tests for monitoring soil quality. In: DORAN, J.W.; JONES, A.J. (Ed.). Methods for assessing soil quality. Madison: Soil Science Society of America, 1996, p.123-141 (SSSA Special publication, 49)

BERTOL, I.; ALBUQUERQUE, J.A.; LEITE, D.; AMARAL A.J.; ZONDAN JR., W.A. Propriedades físicas do solo sob preparo convencional e semeadura direta em rotação e sucessão de culturas, comparadas às do campo nativo. Revista Brasileira de Ciência do Solo, v.28, p.155-163, 2004.

BEUTLER, A.N.; SILVA, M.L.N.; CURI, N.; FERREIRA, M.M.; CRUZ, J.C.; PEREIRA $F^{\circ}$, I.A. Resistência à penetração e permeabilidade de Latossolo Vermelho Distrófico típico sob sistemas de manejo na região dos Cerrados. Revista Brasileira de Ciência do Solo, v.25, n.1, p.167-177, 2001.

CADY, F.B. Experimental design and data management of rotation experiments. Agronomy Journal, v.83, p.50-56. 1991.

CÂMARA, R.K; KLEIN, V.A. Escarificação em plantio direto como técnica de conservação do solo e da água. Revista Brasileira de Ciência do Solo, v.29, p.789-796. 2005.

COMISSÃO DE FERTILIDADE DO SOLO - RS/SC. Recomendação de adubação e de calagem para os Estados do Rio Grande do Sul e de Santa Catarina. 3.ed. Passo Fundo: CFS-NRS/SBCS, 1995. 223p.

COSTA, F.S.; ALBUQUERQUE, J.A.; BAYER, C.; FONTOURA, S.M.V.; WOBETO, C. Propriedades físicas de um Latossolo Bruno afetadas pelos sistemas plantio direto e preparo convencional. Revista Brasileira de Ciência do Solo, v.27, p.527-535, 2003.

DA ROS, C.O.;SECCO, D.; FIORIN, J.E.; PETRERE, C.; CADORE, M.A.; PASA, L. Manejo do solo a partir de campo nativo: efeito sobre a forma e estabilidade da estrutura ao final de cinco anos. Revista Brasileira de Ciência do Solo, v.21, p.241247, 1997.

DENARDIN, J.E.; KOCHHANN, R.A. Requisitos para a implantação e a manutenção do sistema plantio direto. In: CNPT-EMBRAPA, FUNDACEP, FUNDAÇÃO ABC. Plantio direto no Brasil. Passo Fundo: Aldeia Norte, 1993. p.19-27.

DERPSCH, R.; SIDIRAS, N.; ROTH, C.H. Results of studies made from 1977 to 1984 to control erosion by cover crops and no-tillage techniques in Paraná, Brazil. Soil \& Tillage Research, v.8, p.253-263. 1986.

DEXTER, A.R. Amelioration of soil by natural processes. Soil \& Tillage Research, v.20, p.87-100. 1991.

EMBRAPA. CENTRO NACIONAL DE PESQUISA DE SOLOS. Manual de métodos de análise de solo. 2.ed. Brasília: SPI, 1997. 212p. (Embrapa Solos. Documentos, 1)

LLANILLO, R.F.; RICHART, A.; TAVARES FILHO, J.; GUIMARÃES, M.F.; FERREIRA, R.R.M. Evolução de propriedades físicas do solo em função dos sistemas de manejo em culturas anuais. Semina: Ciências Agrárias, v.27, p.205-220, 2006.
KLEIN, V.A.; CÂMARA, R.K. Rendimento de soja e intervalo hídrico ótimo em latossolo vermelho sob plantio direto escarificado. Revista Brasileira de Ciência do Solo, v.31, p.221-227, 2007.

KLEIN, V.A.; LIBARDI, P.L. Faixa de umidade menos limitante ao crescimento vegetal e sua relação com a densidade do solo ao longo do perfil de um latossolo roxo. Ciência Rural, v.30, n.6, p.959-964, 2000.

MARCOLAN, A.L.; ANGHINONI, I. Atributos físicos de um argissolo e rendimento de culturas de acordo com o revolvimento do solo em plantio direto. Revista Brasileira de Ciência do Solo, v.30, p.163-170, 2006.

MARCOLIN, C.D. Propriedades físico-hídrico-mecânicas de solos sob plantio direto, na região de Passo Fundo-RS. 2006. 92f. Dissertação (Mestrado) - Universidade de Passo Fundo, Passo Fundo, 2006.

OLIVEIRA, G.C.; DIAS JR., M.S.; RESCK, D.V.S.; CURI, N. Caracterização química e físico-hídrica de um Latossolo Vermelho após vinte anos de manejo e cultivo do solo. Revista Brasileira de Ciência do Solo, Viçosa, v.28, p.327336, 2004.

REICHERT, J.M.; REINERT, D.J.; BRAIDA, J.A. Qualidade dos solos e sustentabilidade de sistemas agrícolas. Ciência \& Ambiente, v.27, p.29-48, 2003.

SA, M.A.C; LIMA, J.M.; SILVA, M.L.N.; DIAS JR., M.S. Comparação de métodos para o estudo da estabilidade de agregados em solos. Pesquisa Agropecuária Brasileira, v.35, p.1.825-1.834, 2000.

SANTOS, H.P.; TOMM, G.O. Disponibilidade de nutrientes e teor de matéria orgânica em função de sistemas de cultivo e de manejo de solo. Ciência Rural, v.33, p 477-486, 2003.

SECCO, D.; DA ROS, C.O.; SECCO, J.K.; FIORIN, J.E. Atributos físicos e produtividade de culturas em um Latossolo Vermelho Argiloso sob diferentes sistemas de manejo. Revista Brasileira de Ciência do Solo, v.29, p.407-414, 2005.

SPERA, S.T.; SANTOS, H.P.; TOMM, G.O.; FONTANELI, R.S. Efeitos de sistemas de produção de grãos envolvendo pastagens sob plantio direto nos atributos físicos e na produtividade. Revista Brasileira de Ciência do Solo, v.28, p.533-542, 2004.

SPERA, S.T.; SANTOS, H.P.; FONTANELI, R.S.; TOMM, G.O. Efeito de pastagens de inverno e de verão em características físicos do solo, sob plantio direto. Ciência Rural, v.36, p.1.1931.200, 2006.

STATISTICAL ANALYSIS SYSTEMS INSTITUTE. SAS system for Microsoft Windows. version 8.2. Cary, 2003.

STEEL, G.D.; TORRIE, J.H. Principles and procedures of statistics: a biometrical approach. 2.ed. New York: McGrawHill, 1980. 633p. 
STONE, L.F.; SILVEIRA, P.M. Efeitos do sistema de preparo e da rotação de culturas na porosidade e densidade do solo. Revista Brasileira de Ciência do Solo, v.25, p.395-401, 2001.

STRECK, E.V.; KÄMPF, N.; DALMOLIN, R.S.D.; KLAMT, E.; NASCIMENTO, P.C.; SCHNEIDER, P. Solos do Rio Grande do Sul. Porto Alegre: EMATER/RS, UFRGS, 2002. 126p.

TREIN, C.R.; COGO, N.P.; LEVIEN, R. Métodos de preparo do solo na cultura do milho e ressemeadura do trevo, na rotação aveia + trevo/milho, após pastejo intensivo. Revista Brasileira de Ciência do Solo, v.15, p.105-111, 1991. 